\title{
Current applications of high-frequency ultrasonography in dermatology
}

\author{
Adriana Polańska1 , Aleksandra Dańczak-Pazdrowska², Magdalena Jałowska², Ryszard Żaba' ${ }^{1}$ Zygmunt Adamski ${ }^{2}$
}

'Department of Dermatology and Venereology, Poznan University of Medical Sciences, Poznan, Poland 2Department of Dermatology, Poznan University of Medical Sciences, Poznan, Poland

Adv Dermatol Allergol 2017; XXXIV (6): 535-542

DOI: https://doi.org/10.5114/ada.2017.72457

\begin{abstract}
The era of ultrasounds in dermatology started in 1979, when pioneering use of $15 \mathrm{MHz}$ by Alexander and Miller in measuring the skin thickness was introduced. Since then, some new applications of high-frequency ultrasonography (HF-USG) have emerged providing the clinicians with an extra hand in their everyday practice. The main advantages of HF-USG include the possibility of real-time imaging, measurements of morphological and physiological aspects of the skin, safety associated with the use of non-ionizing media as well as the lack of contraindications to its performance. Currently the main clinical use of HF-USG in dermatology regards preoperative assessment of the depth of invasion in melanomas and basal cell carcinomas. The ultrasound image analysis allows noninvasive follow-up of inflammatory skin diseases, like atopic dermatitis, psoriasis and may be used for monitoring effectiveness of therapy in skin lymphomas and sclerotic skin diseases.
\end{abstract}

Key words: high-frequency ultrasonography, basal cell carcinoma, melanoma, atopic dermatitis, mycosis fungoides, hypokeratosis.

\section{Introduction}

In the recent years a great progress has been made in diagnostic methods used in dermatology. Researchers have gained the possibility to examine skin with novel advanced high resolution imaging techniques, including reflectance confocal microscopy and optical coherence tomography $[1,2]$. Although the use of the above techniques in clinical and experimental dermatology is highly promising, especially for the quasi-microscopic characterization of skin tumors, still the skin ultrasonography (USG), as one of the oldest visualizing method in dermatology, cherishes an unflagging popularity as an in vivo rapid and relatively cheap method of examination $[3,4]$. The possibility of real-time imaging, measurements of morphological and physiological aspects of the skin, safety associated with the use of non-ionizing media as well as the lack of contraindications to its performance, are the other advantages of skin sonography.

The pioneering use of waves of $15 \mathrm{MHz}$ in dermatology was introduced by Alexander and Miller in 1979, to measure the thickness of the skin [3]. Since then, some new applications have emerged providing the clinicians with an extra hand in their everyday practice. In this paper we sum up the current applications for high-frequen- cy ultrasonography (HF-USG) in clinical practices on the basis of our 10 years of experience, as well as literature data. We describe both the underlying principles of this method and present its advantages and possible limitations in regards to well-defined applications.

\section{Back to the basic principles of ultrasound...}

The term HF-USG was proposed for frequencies equal to or above $20 \mathrm{MHz}$, which are dedicated to depict the skin and enables to differentiate the structures of less than $100 \mu \mathrm{m}$ on the beam axis (an axial resolution) and $200 \mu \mathrm{m}$ on the scan axis (lateral resolution) [5-8]. While $20 \mathrm{MHz}$ is a well-established method in dermatology used for scanning the whole skin (papillary, reticular dermis and upper parts of subcutaneous tissue - depending on the localization), higher resolutions in the range up to $100 \mathrm{MHz}$ will allow for much more detailed imaging of the epidermis and the upper dermis - with an axial resolution of approximately $10 \mu \mathrm{m}$ and lateral $84 \mu \mathrm{m}$ (however such probes are still used experimentally). The detection of lesions located within the lower parts of subcutaneous tissue, as well as for examination of regional lymph nodes, needs greater penetrations of

Address for correspondence: Adriana Polańska MD, PhD, Department of Dermatology and Venereology, Poznan University of Medical Sciences, 49 Przybyszewskiego St, 60-355 Poznan, Poland, phone: +48 516123 858, e-mail: adriana-polanska@wp.pl Received: 12.10 .2016 , accepted: 8.11.2016. 
the wave to these structures, which are possible with 5 to $12 \mathrm{MHz}$ [5].

It is worth emphasizing that the USG image depends on the tissue composition, which influences the properties of the reflected sound wave. Some waves falling on the boundary of the two media with different acoustic properties are reflected [5-9]. They return to the transducer as an echo with a certain energy. Other waves penetrate deeper to e.g. find the next border or to be muted. Ultrasound image is therefore a reflection of acoustic impedance of divers centers, which is the exponent of the resistance that puts a tissue propagation and which depends on the density and elasticity of the resort. The greater the difference in acoustic impedance, the more the wave is reflected. The biggest differences are between soft tissue and bone or calcification - where we may observe a very strong echo and acoustic shadow [5-8].

Presentation of type B (brightness) is the most commonly used in dermatological applications and allows the display of a set of points in the form of two-dimensional image [6]. Echo returning to the transducer is converted to dots. Their width corresponds to the echo at its base, and the brightness to the received amount of energy. So the more the waves were reflected, the more this spot is brighter. Finally, the amount of returning energy is a function of time and is encoded in a color scale ranging from 0 to $255[5,7]$. Regardless of the encoding method, black areas are referred to as an anechoic one, while white areas as a hyperechoic one. To illustrate the skin in the presentation of type $B$, the linear probes were used. Additionally some ultrasound devices enable three-dimensional image reconstruction (3D) $[6,7]$.

\section{How the healthy skin looks like}

An ultrasound image of healthy skin is divided into 3 layers [5-11]:

- entrance echo (entry echo) - highly echogenic band,

- dermis (often with the deeper layers of the epidermis) - layer rich in dispersed echoes of varying intensities,

- the subcutaneous tissue - an anechoic or low echogenicity layer in which it is sometimes possible to observe the structures with enhanced echogenicity related to the presence of septae.

It is debatable whether the entry echo corresponds to the full thickness of the epidermis or it is only related to the thickness of stratum corneum. Some authors suggest using the term "echo from the epidermis" $[8,10]$. In Cammarota et al.'s opinion, entry echo is produced by the differences of the acoustic impedance between gel and the skin [12]. According to Seidenari, entry echo contains the upper part of epidermis which is related to the water/stratum corneum interface, while the echo-rich line beneath corresponds to stratum corneum/stratum Malpighi interface [12]. It is believed that this double entry echo (bilaminar according to Wortsman) is more likely to be detected within localizations with thick stratum corneum, like palms and soles [13].

In previous studies there were no correlations between the thickness of the entry-echo and histologically measured thickness of the stratum corneum or full-thickness skin [8]. Both structures (layer Malpighi and papillary skin) have similar echogenicity (a result of similar acoustic properties) and with the use of $20 \mathrm{MHz}$ transducer it appears that there is no possibility of varying the different layers of the epidermis (beyond the stratum corneum), or to visualize the dermo-epidermal junction [8, 9].

Below the entry echo there is a less echogenic layer, with the thickness varying depending on the anatomical locations from 1 to $2.5 \mathrm{~mm}$, which expresses the dermis and possibly the lower layers of the epidermis [7, 12]. This part is characterized by heterogeneous echogenicity. In general the upper parts (papillary dermis) are less echogenic than lower (reticular layer), what is related to the tighter packing of thick parallel arranged bundles of collagen in deeper layers of the skin [9, 12]. Additionally, within the dermis hair follicles can be observed, which are hypoechogenic structures.

Olsen et al. revealed the differences in skin thickness in regards to sex and localization. Female skin is thinner than male skin and extremity skin is thinner than truncal skin [14]. Also echogenicity of the dermis varies as a function of age. It is the lowest in newborns, then enhances after a few months of life to a progressive increase in adult individuals $[12,14,15]$.

The echogenicity of the dermis is influenced by several factors, like orientation of collagen fibers, type of ground substance and water content [5-12]. Any pathological conditions associated with the accumulation of fibers in the skin will therefore cause an increase in its echogenicity. And on the other hand - damage for example of elastic fibers, increased numbers of cells (inflammatory, neoplastic) or enhanced water content will be responsible for lowering the total echogenicity of this layer. For inflammatory dermatoses (psoriasis, eczema, atopic dermatitis) as well as in mycosis fungoides, similarly to the skin elastosis, subepidermal low echogenic band (SLEB) is easy to detect $[16,17]$.

The phenomenon detected within sun-exposed skin underneath the entry echo in elderly individuals is the presence of the subepidermal anechogenic or low echogenic band known as SLEB. The SLEB was first reported in 1989 by de Rigal et al. and its origin is not well understood, probably it is derived from skin elastosis and accumulation of glycosaminoglycans, which possess increased water-binding capacity $[9,12]$. Due to the fact that SLEB can be observed within the skin exposed to sun, it may serve as a marker of photodamage and can be used to demonstrate the efficacy of anti-aging preparations [9, 12, 14, 15]. Sandby-Møller et al. reported that SLEB increased significantly with age at both the forehead and at the dor- 
sal aspect of the forearm, and was present in over $50 \%$ of adults above the age of 40 years [18].

The subcutaneous tissue is a structure of lack/low echogenicity, hence the boundary between the dermis and hypodermis is easy to visualize. Occasionally, in the subcutaneous tissue, we can see septae dividing the body fat, as well as fascia and vessels, and in specific locations, such as the forehead or nose, we can also observe the surfaces of the bones [7, 12].

\section{Skin malignancies}

Currently the main clinical use of HF-USG in dermatology regards preoperative assessment of the depth of invasion in melanomas and basal cell carcinomas (BCC). Especially in melanomas, preoperative determination of the surgical margin determines the future management and patient's prognosis. Ultrasound of BCC and melanoma present similarly - as hypoechoic circular or oval shape structures surrounded by hyperechoic areas [12, 13, 19] (Figures 1, 2). This makes the use of HF-USG in the final diagnosis of the type of the tumor not completely possible. Also differentiation between the benign and malignant tumors on the ultrasound pattern is unlikely, although some authors postulate that benign tumors as well as BCC present more internal echoes than melanomas $[12,20]$. Additionally, in some cases reported below, USG image analysis of skin tumors may show features which facilitates recognition $[12,19,20]$.

Usually the boundary between hypoechoic infiltration of cancer and hyperechoic dermis is sharp and easy to observe, hence it is possible to determine the dimensions of the tumor. Only in the case of tumors which cross the border of the dermis and penetrate into the subcutaneous tissue (that is hypoechoic), the depth of infiltration cannot be assessed [8-13].

\section{Sonograms of non-melanoma skin cancers (NMSC)}

In BCC, increased entry echo can be visualized, while in the case of concomitant erosions and small ulcers its break is detected [9-12]. Additionally, some researchers described the strengthening of the echo within the dermis underlying the tumor, particularly in nodular BCC $[12,13,20]$. Furthermore, in some subtypes of BCC within the hypoechoic tumor in homogeneously scattered hyperechoic, fine structures known as "flower cotton" can be observed [21]. These phenomena probably respond to calcifications, cornified cysts, nests of apoptotic or parakeratotic cells as well as cell necrosis [7, 8, 12]. Not all BCC have sharply defined borders, it is estimated that $30-70 \%$ of BCC present a well-defined base border, while $50 \%$ of them is sharply demarcated laterally [12, 20]. Most difficulties in exact assessment of tumor margins regards morpheaform and infiltrative types of BCC. The hyperplasia of sebaceous glands especially on the nose presenting as blurriness (blurry tumor) may also contribute to the difficulty in image interpretation [13]. Important limitation in establishing BCC size is concomitant presentation of elastosis within sun-exposed areas, which as mentioned above, also shows decreased echogenicity [7-9].

Due to the presence of scales or crusts on the surface of squamous cell carcinoma, we can often observe shadows running perpendicularly to the surface of the skin,

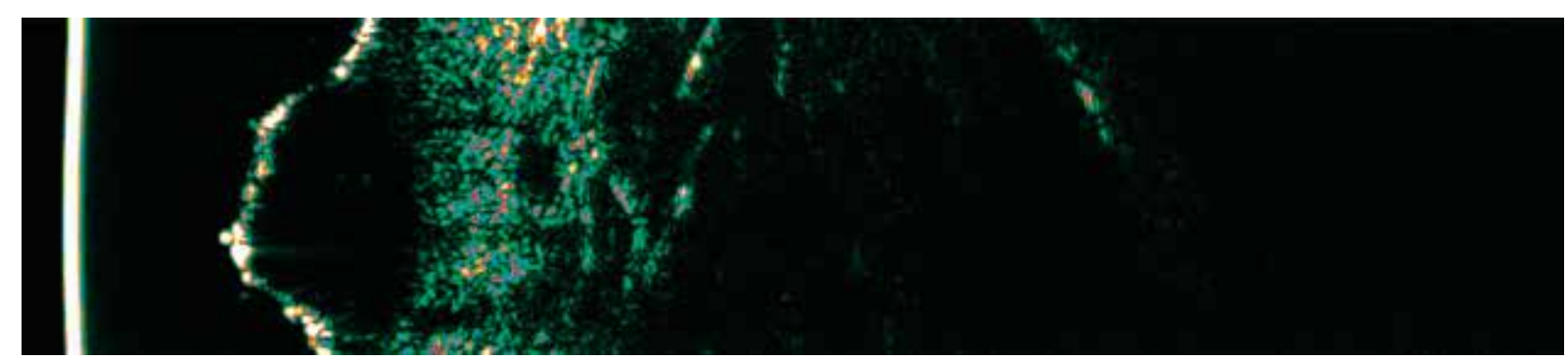

Figure 1. Basal cell carcinoma on the neck

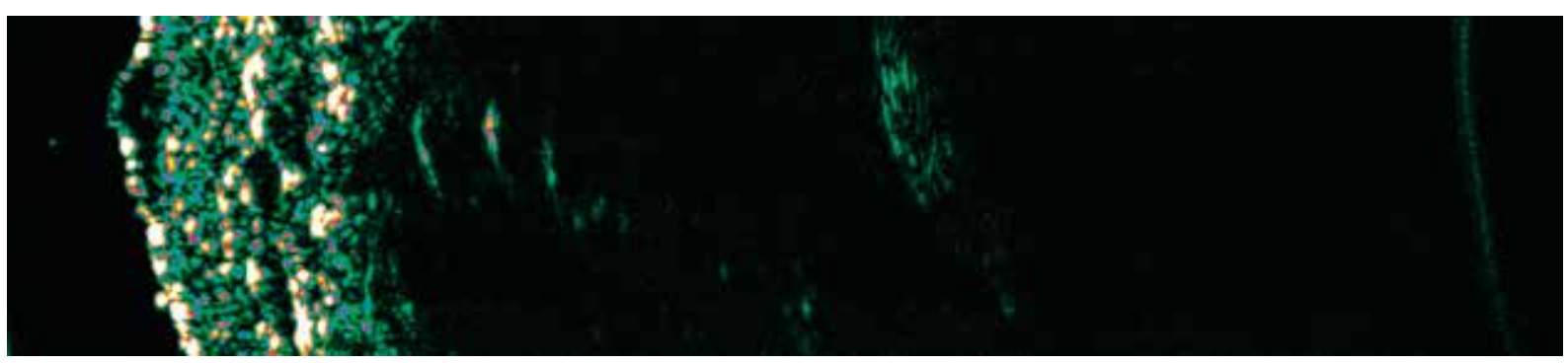

Figure 2. Melanoma superficiale - the right forearm of a 64-year-old woman 
which in effect may prevent making correct analysis of the image $[12,20]$.

\section{Sonograms of melanoma}

Typically in melanomas, homogenous echo-lucent areas can be observed, which is the opposite to BCC $[8,11$, $12,20]$. The shape of this area depends on the subtype, where the nodular form may have a spherical arrangement, while in superficial spreading melanoma, the echolucent area is reported as a thin layer located parallel to the entry echo (Figure 2). However, there are no ultrasonographic features pathognomonic for melanoma. It is therefore impossible to differentiate melanoma from melanocytic nevi. The latter present themselves as anechoic structures like melanomas $[3,5,12]$. The analysis of the USG image by Harland et al. (with detailed assessment of acoustic shadowing and entry echo line enhancement) revealed that HF-USG could distinguish melanoma from seborrheic keratosis (basal cell papillomas) with 100\% sensitivity and $79 \%$ specificity, while differentiation between melanomas and melanocytic nevi using this method is poor (30\% specificity) [22]. Seborrheic keratosis presents marked attenuating properties (acoustic shadow) and enhanced entry echo due to the hyperkeratosis, what is in contrast to melanomas, where little shadowing and usually thin entry echo is observed [22].

\section{Other oncological applications of HF-USG}

Another use of ultrasound in skin oncology, which is worth mentioning, is the ability to visualize the thickness of the neoplastic infiltration in skin lymphomas. In mycosis fungoides, within lesions, the hypoechoic band is visible just below the entry echo (SLEB), which thickness may correspond to the severity of the disease. The SLEB thickness measurement can also be used to monitor the effects of the therapy applied [17, 23].

\section{Correlation between histologic and sonometric parameters in skin tumors}

From the clinical point of view, the agreement between ultrasound and histological measurements in relation to the depth of tumor invasion in melanoma and BCC is well documented. Many studies have confirmed the high correlation between the two methods of up to $88 \%$ and $96 \%$ (correlation coefficient) [19, 24-28]. This is particularly important in planning the extent of the surgery. Good knowledge of the limits of infiltration may contribute to an increased percentage of radical treatments. It is known, however, that the thickness of the tumor determined by ultrasound is slightly greater than the thickness determined histologically $[12,20]$. This difference is due to the shrinkage of the tissue during histological preparation and the presence of inflammatory infiltrate around the tumor (with ultrasound resolution insufficient to distinguish malignant infiltration from in- flammatory one). In BCC, this inflammation underneath the tumor mass (made of dilated vessels and giant cell infiltration) is called "angles of the bottom" [13].

Desai et al. confirm that skin sonography may improve surgical precision in BCC. They assessed fifty superficial and nodular BCC with a good correlation between clinical and ultrasonic measurements in terms of width, depth, and length [29]. In another study where Mohs surgery was used in BCC and SCC in relation to tumors with clinical areas greater than the median of $1.74 \mathrm{~cm}^{2}$ sensitivity of ultrasound was $80 \%$ and specificity $84 \%$, however HF-USG failed to detect subclinical tumor extensions with accuracy [30].

The disagreement between sonometric and histopathological measurements in melanomas accounts for tenths of a millimeter. Hoffmann et al. examined 55 melanomas and found sonometric values of melanoma greater by $0.4 \mathrm{~mm}$ in comparison to histometry [20]. The overestimation of the melanoma size may be caused by dense infiltrate of lymphocytic infiltration (related to regression phenomenon) or by dermal nevus components underneath the melanoma tissue as well [19, 31]. Additionally the shrinkage of the biopsied material is not without significance.

Very thin melanomas cannot be detected with $20 \mathrm{MHz}$ and for such tumors with the thickness less than $1 \mathrm{~mm}$, higher frequencies were also evaluated [7]. Hayashi et al. used $30 \mathrm{MHz}$ USG and reported a good correlation between sonographic and histologic thickness $(r=0.89)$, while in melanomas affecting soles the correlation was even higher $(r=0.94$ [31]). Pellacani and Seidenari in 2003 proposed an algorithm based on echographic, clinical and videomicroscopic criteria for preoperative evaluation of melanoma thickness and differentiation between thin and thick melanomas. They recommend the use of HF-USG $(20 \mathrm{MHz})$ as the first step in this algorithm [19]. In 2008 Guitera et al. proposed $75 \mathrm{MHz}$ scanner in invasive melanomas with high concordance with histometry $(r=0.908)[27]$.

\section{Inflammatory diseases of the skin}

In inflammatory skin diseases (eczema, atopic dermatitis (AD), psoriasis), SLEB of varied thickness and decreased echogenicity of the other layers of the dermis may be shown (Figure 3) [7, 8, 32, 33]. The lower echogenicity of the skin is mainly due to swelling of the skin and inflammatory cell infiltration. The skin edema, due to the presence of water, grows the distance between the collagen fibers resulting in decreased density of the tissue. Areas of reduced echogenicity can be observed in both the upper and lower layers of the dermis, what is largely dependent on the triggering cause. In eczema, as well as in chronic venous insufficiency (stasis dermatitis), localized hypoechoic areas primarily in the upper layers of the dermis can be visualized, while the swelling 


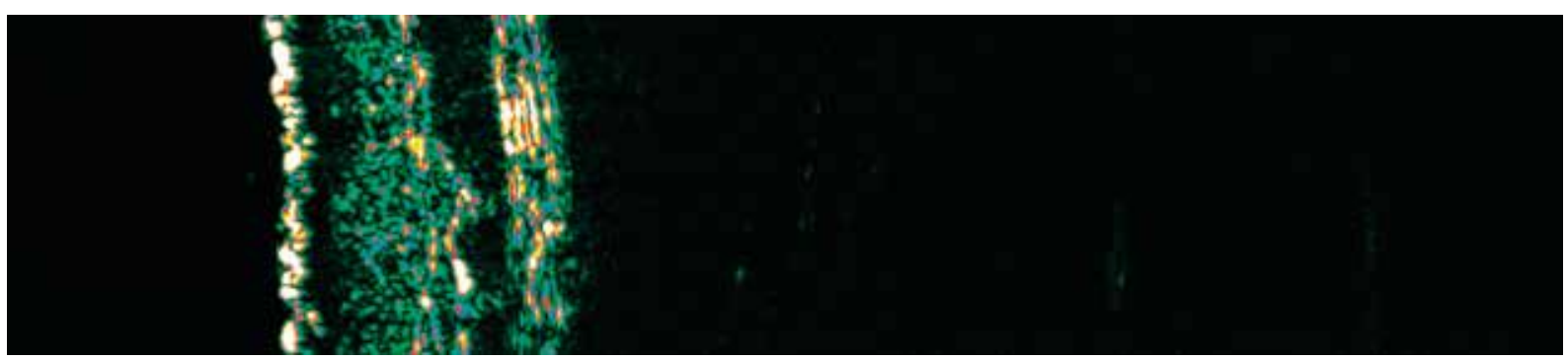

Figure 3. Subepidermal low echogenic band in a 26-year-old patient suffering from atopic dermatitis

caused by congestive heart failure is primarily located within the lower part of the reticular layer, adjacent directly to the subcutaneous tissue [34].

A characteristic feature of the sonogram of $A D$ is the presence of SLEB, which may be accompanied by lower, as compared to the healthy skin, echogenicity of the other layers of the skin $[9,12,14,15,32]$. The SLEB thickness measurement correlates with the severity of skin lesions in $A D$ and can be used as an objective parameter monitoring the course of disease [35-37]. We compared the sonograms of AD patients with histopathological examination and found statistically significant correlations between the hypoechoic band thickness and the following parameters: the degree of epidermal hyperplasia, the degree of epidermal hyperkeratosis, the degree of parakeratosis and the degree of spongiosis as well as the intensity of inflammatory infiltrates. Skin echogenicity strongly correlated negatively with the intensity of inflammatory infiltrates [35]. Moreover, in some patients with $A D$ within healthy skin around the affected region, we could detected also thin SLEB. It seems that this phenomenon may be related to the presence of subclinical inflammation [32].

An ultrasound image of psoriatic plaque presents itself similarly to AD. In addition to the SLEB, in psoriasis we may detect thickened entry echo and streaky, perpendicular to the entry echo shadows caused most likely by air bubbles trapped between the scales [9]. Furthermore, the total thickness of the skin within psoriatic plaques is increased and its reduction during the therapy can be objectively shown [38].

Another documented application of ultrasound in dermatology is the evaluation of the patch test. Sono- graphic analysis of patch test results revealed that an increase in skin thickness (related to the severity of skin swelling) was related to the intensity of the allergic reaction [12] (Figure 4).

One of the newest utilization of USG in dermatology is ultrasound staging and diagnosis of hidradenitis suppurativa [39, 40]. Wortsman proposed sonographic scoring of hidradenitis suppurativa based on the amount and differentiation of sonographic main type lesions (pseudocysts, fluid collection, fistulous tracks). The ultrasound image analysis was able to detect the large number of subclinical lesions that could not be reliably discriminated through palpation only [13, 39].

\section{Diseases of the skin thickening}

One of the earliest applications of HF-USG is the monitoring of the therapeutic effect of diseases associated with excessive accumulation of collagen in the skin [41]. An increase in depositions in collagen fibers enhances the echogenicity of the skin and its thickness. In the case of systemic sclerosis, skin thickness was measured within the phalanges (mainly distal and middle), and its thickening as compared to the control group was demonstrated [41, 42]. In morphea or the sclerodermic variant of chronic graft versus host disease (GVHD), a clinically noticeable tightening of skin appears, which on sonograms presents itself as an increase in skin density [43]. It is worth noting that in the case of these diseases, a significant impact on the ultrasound image relies on the clinical phase of the disease. Increased echogenicity and thickening of the skin is visible only during the sclerotic phase, while in the inflammatory phase, a reduction in its echo-

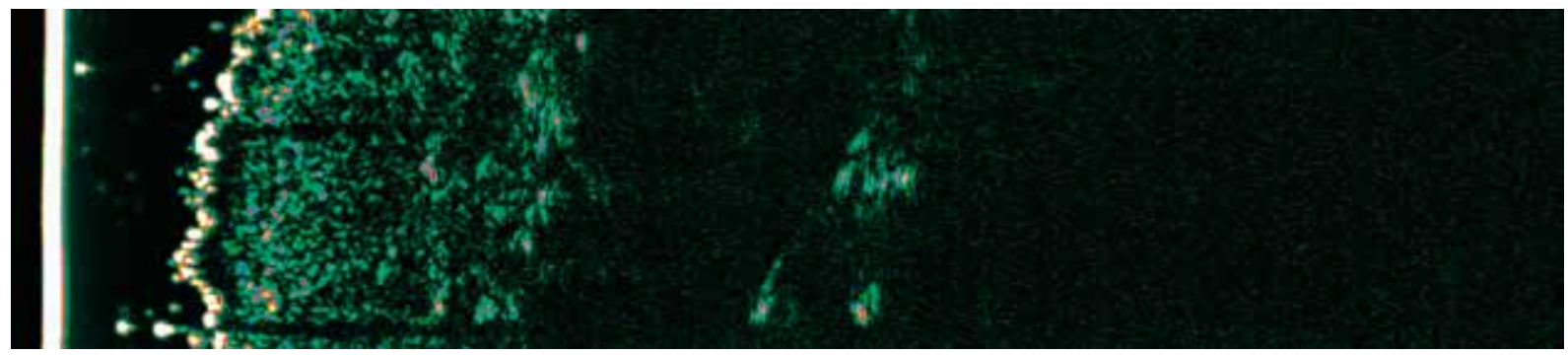

Figure 4. A vesicle in patch tests - positive patch test result towards nickel sulfate 


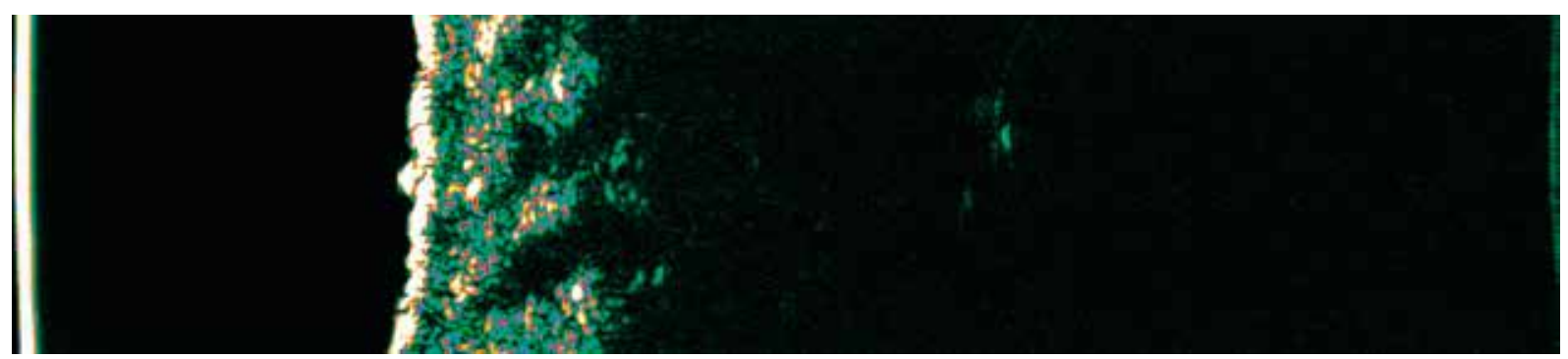

Figure 5. Cellulite - posterior surface of the left hip in a 45-year-old woman

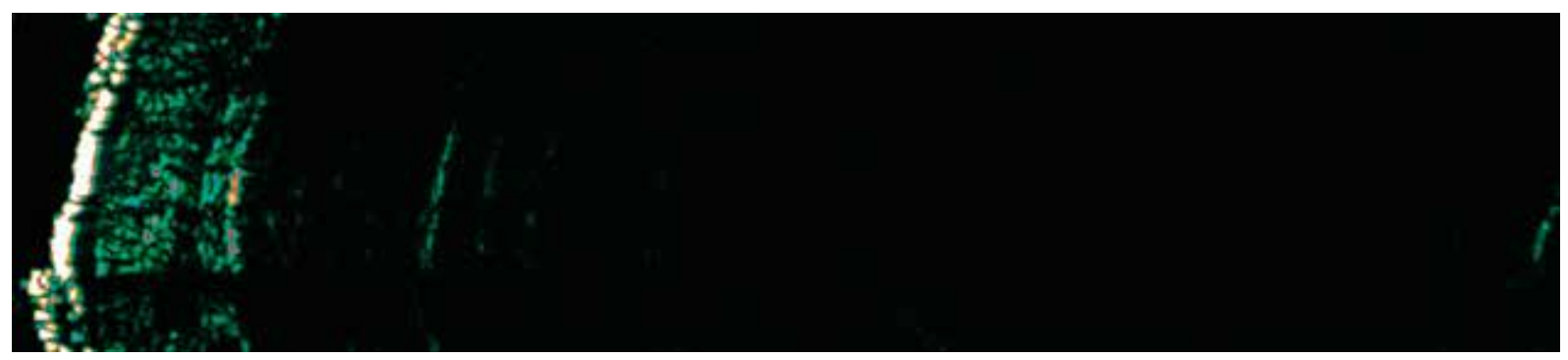

Figure 6. Circumscribed palmar hypokeratosis

genicity can be observed. On the other hand, the atrophic phase is characterized by the thinning of the skin which echogenicity is similar to the surrounding tissue. Image analysis is complicated by the fact that inflammatory and sclerotic phases often coexist even within the same plaque. It seems, therefore, that the thickness of the skin in such diseases is a more important parameter than the assessment of its echogenicity.

\section{Cosmetic applications}

As it was mentioned at the beginning, the measurement of skin thickness, especially the analysis of SLEB width within the sun-exposed areas, contribute to the use of HF-USG in anti-aging medicine. The detection and identification of cosmetic fillers seem to be another spreading application of HF-USG [15]. A typical USG image of hyaluronic acid deposits is round or oval-shaped, anechoic pseudocysts. With the use of HF-USG it is possible to perform ultrasound-guided hyaluronidase injections and visualize the decrease in the size of the hyaluronic acid deposits [44]. Similarly to hyaluronic acid injections, also injections of botulinum toxin A can be visualized sonographically, but in this case they produce an increase in subcutaneous echogenicity with blurriness at the border between subcutaneous and muscle tissue $[13,45]$.

The use of HF-USG gives the opportunity for the monitoring of anticellulite therapy [46, 47]. Cellulite appears as rough ingrowths of subcutaneous tissue into the dermis what may resemble saw teeth. The treatment of cellulite provokes smoothing the border between skin and subcutaneous tissue and reduction of strands penetrating the dermis [48, 49] (Figure 5).

\section{Other}

The use of HF-USG was reported in the assessment of post steroid skin atrophy $[9,12,50]$. Reduced skin thickness by up to $40 \%$ was observed after an intradermal injection of $0.1 \mathrm{ml}$ of a triamcinolone acetonide suspension in the course of 4-8 weeks [9, 12]. The potency of glucocorticosteroids in regards of their atrophogenicity was evaluated with HF-USG. It was revealed that prednicarbate and hydrocortisone aceponate ointments produced a similar decrease in skin thickness as corresponding vehicles [50].

Other practical applications of high-frequency ultrasound is the monitoring of venous microsclerotherapy [51]. A prick of the blood vessel can be performed under ultrasound control and the vessel diameter can be evaluated after surgery.

Moreover, HF-USG may serve as a useful tool in recognition of rarely diagnosed disease - circumscribed palmar hypokeratosis - where characteristic ultrasonographic features were firstly described by us. Within the affected skin we can observe single increased entrance echo below which SLEB is detected. The border between lesional and nonlesional skin is typically sharply accentuated with enhancement of skin echogenicity. The non-affected skin, at the border, presented typical double entrance echo (Figure 6).

\section{Conclusions}

Ultrasound examination using high frequencies is surprisingly an underappreciated method among many used in dermatology. It is worth noting that, for example, in Germany the ultrasound examination is reimbursed by 
the national health insurance in the case of melanoma, and learning skin sonography is an important element of training during specialization. It seems that the routine use of ultrasound would be particularly useful: (1) in skin neoplasms including BCC and melanoma, (2) in order to objectify the assessment of the effects of dermatological treatment and in the field of so-called aesthetic dermatology.

\section{Conflict of interest}

The authors declare no conflict of interest.

\section{References}

1. Rajadhyaksha M, Gonzales S, Zavislan JM, et al. In vivo confocal scanning laser microscopy of human skin II: advances in intrumentation and comparison with histology. J Invest Dermatol 1999; 113: 293-303.

2. Hinz T, Ehler LK, Hornung T, et al. Preoperative characterization of basall cell carcinoma comparing tumor thickness measurement by optical coherence tomography, $20 \mathrm{MHz}$ ultrasound and histopathology. Acta Derm Venereol 2012; 92: 132-7.

3. Fornage BD. High-frequency sonography of the skin. Eur J Ultrasound 1995; 2: 173-82.

4. Białynicki-Birula R. New possibilities of using ultrasound high-frequency $(22 \mathrm{MHz})$ in dermatology. Habilitation thesis. Wrocław 2014.

5. Shmid-Wendter MH, Dill-Muller D. Ultrasound technology in dermatology. Sem Cutan Med Surg 2008; 27: 44-51.

6. Dill-Müller D, Maschke J. Ultrasonography in dermatology. J Dtsch Dermatol Ges 2007; 5: 689-707.

7. Jasaitiene D, Valiukeviciene S, Linkeviciute G, et al. Principles of high-frequency ultrasonography for investigation of skin pathology. J Eur Acad Dermatol Venereol 2011; 25: 375-82.

8. Jemec GB, Gniadecka M, Ulrich J. Ultrasound in dermatology. Part I: High frequency ultrasound. Eur I Dermatol 2000; 10: 492-7.

9. Fornage B. High-frequency ultrasonography of the skin. Radiology 1993; 189: 69-76.

10. Hoffmann K, Dirschka T, Schwarze H, et al. $20 \mathrm{MHz}$ sonography, colorimetry and image analysis in the evaluation of psoriasis vulgaris. J Dermatol 1995; 9: 103-10.

11. Cammarota T, Pinto F, Magliaro A, Sarno A. Current uses of diagnostic high-frequency US in dermatology. Eur J Radiol 1998; 27 Suppl 2: S215-23.

12. Seidenari S. High-frequency sonography combined with image analysis: a noninvasive objective method for skin evaluation and desription. Clin Dermatol 1995; 13: 349-59.

13. Wortsman X. Ultrasound in dermatology: why, how and when? Semin Ultrasound CT MRI 2013; 34: 177-95.

14. Olsen L, Takiwaki H, Serup J. High-frequency ultrasound charcterization of normal skin. Skin thickness and echografic density of 22 anatomical sites. Skin Res Technol 1995; 1: 74-80.

15. Waller JM, Maibach HI. Age and skin structure and function, a quantitative approach (I): blood flow, $\mathrm{pH}$, thickness, and ultrasound echogenicity. Skin Res Technol 2005; 11: 221-35.

16. Polańska A, Silny W, Jenerowicz D, et al. Monitoring of therapy in atopic dermatitis - observations with the use of high-frequency ultrasonography. Skin Res Technol 2015; 21: 35-40.
17. Olek-Hrab K, Silny W, Dańczak-Pazdrowska A, et al. Ultraviolet A1 phototherapy for mycosis fungoides. Clin Exp Dermatol 2013; 38: 126-30.

18. Sandby-Møller J, Thieden E, Philipsen PA, et al. Dermal echogenicity: a biological indicator of individual cumulative UVR exposure? Arch Dermatol Res 2004; 295: 498-504.

19. Pellacani G, Seidenari S. Preoperative melanoma thickness determination by 20-MHz sonography and digital videomicroscopy in combination. Arch Dermatol 2003; 139: $293-8$.

20. Hoffmann, K, el-Gammal, S, Winkler, K et al. Skin tumours in high-frequency ultrasound. In: Altmeyer P, el-Gammal S, Hoffmann K (eds.). Ultrasound in dermatology. SpringerVerlag, Berlin/Heidelberg/New York 1992; 181-201.

21. Wortsman X, Jemec GBE. High resolution ultrasound applications in dermatology. Rev Chilena Dermatol 2006; 22: 37-45.

22. Harland CC, Kale SG, Jackson P, et al. Differentiation of common benign pigmented skin lesions from melanoma by high-resolution ultrasound. Br J Dermatol 2000; 143: 281-9.

23. Polańska A, Osmola-Mańkowska A, Olek-Hrab K, et al. Do we need high-frequency ultrasonography in mycosis fungoides? Abstract book, P1065, EADV 2016 Viena.

24. Tacke J, Haagen G, Hornstein OP, et al. Clinical relevance of sonometry-derived tumour thickness in malignant melanoma: a statistical analysis. Br J Dermatol 1995; 132: 209-14.

25. Lassau N, Koscielny S, Avril MF, et al. Prognostic value of angiogenesis evaluated with high-frequency and color Doppler sonography for preoperative assessment of melanomas. AJR Am J Roentgenol 2002; 178: 1547-51.

26. Serrone L, Solivetti FM, Thorel MF, et al. High frequency ultrasound in the preoperative staging of primary melanoma: a statistical analysis. Melanoma Res 2002; 12: 287-90.

27. Guitera P, Li LX, Crotty K, et al. Melanoma histological Breslow thickness predicted by $75-\mathrm{MHz}$ ultrasonography. Br J Dermatol 2008; 159: 364-9.

28. Bobadilla F, Wortsman X, Muńoz C, et al. Pre-surgical high resolution ultrasound of facial basal cell carcinoma: correlation with histology. Cancer Imaging 2008; 22: 63-72.

29. Desai TD, Desai AD, Horowitz DC, et al. The use of high-frequency ultrasound in the evaluation of superficial and nodular basal cell carcinomas. Dermatol Surg 2007; 33: 1220-7.

30. Jambusaria-Pahlajani A, Schmults CD, Miller CJ, et al. Test characteristics of high-resolution ultrasound in the preoperative assessment of margins of basal cell and squamous cell carcinoma in patients undergoing Mohs micrographic surgery. Dermatol Surg 2009; 35: 9-15.

31. Hayashi K, Koga H, Uhara H, Saida T. High-frequency 30-MHz sonography in preoperative assessment of tumor thickness of primary melanoma: usefulness in determination of surgical margin and indication for sentinel lymph node biopsy. Int J Clin Oncol 2009; 14: 426-30.

32. Dańczak-Pazdrowska A, Polańska A, Silny W, et al. Seemingly healthy skin in atopic dermatitis: observations with the use of high-frequency ultrasonography, preliminary study. Skin Res Technol 2012; 18: 162-7.

33. Rallan D, Harland CC. Ultrasound in dermatology - basic principles and applications. Clin Exp Dermatol 2003; 28: 632-8.

34. Gniadecka M. Dermal edema in lipodermatosclerosis: distribution, effects of posture and compressive therapy evaluated by high-frequency ultrasonography. Acta Derm Venereol 1995; 75: 120-4.

35. Polańska A, Dańczak-Pazdrowska A, Silny W, et al. Comparison between high-frequency ultrasonography (Dermascan 
C, version 3) and histopathology in atopic dermatitis. Skin Res Technol 2013; 19: 432-7.

36. Silny W, Osmola-Mańkowska A, Czarnecka-Operacz M, et al. Wąskozakresowa UVA-1 phototherapy in dermatological treatment - the first Polish experience. Post Alergol Dermatol 2010; 27: 1-10.

37. Osmola-Mańkowska A, Polańska A, Silny W, et al. Topical tacrolimus vs medium-dose ultraviolet A1 phototherapy in the treatment of atopic dermatitis - a preliminary study in relation to parameters of the epidermal barrier function and high-frequency ultrasonography. Eur Rev Med Pharmacol Sci 2014; 18: 3927-34.

38. Gupta AK, Turnbull DH, Harasiewicz KA, et al. The use of high-frequency ultrasound as a method of assessing the severity of a plaque of psoriasis. Arch Dermatol 1996; 132: 658-62.

39. Wortsman X, Rodriguez C, Lobos C, et al. Ultrasound diagnosis and staging in pediatric hidradenitis suppurativa. Pediatr Dermatol 2016; 33: 260-4.

40. Wortsman X. Imaging of hidradenitis suppurativa. Dermatol Clin 2016; 34: 59-68.

41. Tedstone JL, Richards SM, Garman RD, Ruzek MC. UItrasound imaging accurately detects skin thickening in a mouse scleroderma model. Ultrasound Med Biol 2008; 34: 1239-47.

42. Silny W, Osmola-Mańkowska A, Czarnecka-Operacz M, et al. Eosinophilic fascitis: a report of two cases treated with ultraviolet A1 phototherapy. Photodermatol Photoimmunol Photomed 2009; 25: 325-7.

43. Osmola-Mańkowska A, Silny W, Dańczak-Pazdrowska A, et al. Assessment of chronic sclerodermoid graft-versus-host disease patients, using $20 \mathrm{MHz}$ high-frequency ultrasonography and cutometer methods. Skin Res Technol 2013; 19 : 417-22.

44. Wortsman X, Wortsman J, Orlandi C, et al. Ultrasound detection and identification of cosmetic fillers in the skin. J Eur Acad Dermatol Venereol 2012; 26: 292-301.

45. Quezada-Gaon N, Wortsman X, Peńaloza O, Carrasco JE. Comparison of clinical marking and ultrasound-guided injection of botulinum type $A$ toxin into the masseter muscles for treating bruxism and its cosmetic effects. J Cosmet Dermatol 2016 Jan 22. doi: 10.1111/jocd.12208. [Epub ahead of print]

46. Bousquet-Rouaud R, Bazan M, Chaintreuil J, Echague A. High-frequency ultrasound evaluation of cellulite treated with the 1064 nm Nd:YAG laser. J Cosmet Laser Ther 2009; 11: 34-44.

47. Mlosek RK, Dębowska RM, Lewandowski M, et al. Imaging of the skin and subcutaneous tissue using classical and highfrequency ultrasonographies in anti-cellulite therapy. Skin Res Technol 2011; 17: 461-8.

48. Kerscher MJ, Hart H, Korting HC, Stalleicken D. In vivo assessment of the atrophogenic potency of mometasone furoate, a newly developed chlorinated potent topical glucocorticoid as compared to other topical glucocorticoids old and new. Int J Clin Pharmacol Ther 1995; 33: 187-9.

49. Kerscher MJ, Korting HC. Topical glucocorticoids of the nonfluorinated double-ester type. Lack of atrophogenicity in normal skin as assessed by high-frequency ultrasound. Acta Derm Venereol 1992; 72: 214-6.

50. Unholzer A, Korting HC. High-frequency ultrasound in the evaluation of pharmacological effects on the skin. Skin Pharmacol Appl Skin Physiol 2002; 15: 71-84.
51. Mlosek RK, Malinowska S, Serafin-King M, et al. Ultrasound monitoring of the laser closing of blood vessels - own experience. Acta Bio-Optica et Informatica Medica 2011; 17: 276-8. 